\title{
MORTALIDADE EM MUNICÍPIOS DO ESTADO DE SÃO PAULO E INDICADORES SÓCIO- ECONÔMICOS: UMA ANÁLISE DE AGRUPAMENTO
}

\author{
Maria Mercedes Loureiro Escuder ${ }^{1}$, Jorge Kayano ${ }^{2}$, Luiza Sterman \\ Heimann
}

O trabalho discute a análise de dados de mortalidade em municípios do Estado de Săo Paulo agrupados segundo indicadores de natureza sócio-económica como instrumento de investigação epidemiológica no campo do planejamento e gestão de serviços de saúde. Foram extraídos de bancos de dados do IBGE e da Fundaçăo SEADE, 15 indicadores de natureza sócio-económica de todos os 49 municípios com mais de 100 mil habitantes do Estado de São Paulo. Para o reconhecimento de grupos homogêneos de municípios aplicouse a técnica de análise de agrupamento (Cluster Analysis), identificando 7 grupos. Examinouse a seguir a capacidade discriminatória que as condiçőes de saúde teriam para os grupos identificados, através da técnica de análise discriminante, usando o agrupamento identificado como variável dependente e os coeficientes de mortalidade por causa específica como variáveis independentes, ou preditoras de grupo. Essa técnica apontou uma concordância entre valores observados e preditos de $83,67 \%$. Analisando-se as diferenças estatisticamente significantes através da análise de variância, foi possivel classificar qualitativamente os 7 grupos de municípios. Esse exercício demostra a potencialidade da aplicação de técnicas de análise multivariada no reconhecimento da complexa realidade social e sanitária que solicita um projeto de intervenção.

\section{Introdução}

As técnicas de processamento e análise de dados habitualmente empregadas pela epidemiologia na investigação do processo saúde-doença podem constituir instrumentos úteis e versáteis para a administração de serviços de saúde, tanto no que interessa ao reconhecimento da realidade sobre a qual se atua quanto no que diz respeito à definição de estratégias de intervenção.

Vaughan e Morrow publicaram em 1989 um manual de epidemiologia para administradores de serviços de saúde, onde se lê que a gerência racional de serviços de saúde no nível da comunidade demanda o uso criativo de métodos epidemiológicos, sugerindo que,

\footnotetext{
${ }^{1}$ Pesquisadora do Núcleo de Endemias e Epidemias, mestranda da Faculdade de Saúde Pública da Universidade de São Paulo.

${ }_{3}^{2}$ Médico sanitarista, pesquisador do Núcleo de Investigação em Sistemas e Serviços de Saúde.

3 Médica sanitarista, Mestre em Medicina Preventiva pela Faculdade de Medicina da Universidade de São Paulo, coondenadora do Núcleo de Investigaçáo em Sistemas e Serviços de Saúde.

Endereço para correspondência: Instituto de Saúde - Secretaria de Estado da Saúde de São Paulo - Rua Santo Antonio, 590, 4⿳⺈ andar - São Paulo, SP. CEP 01314-000. Fax: 605-2772. Tel: 605-6669
} 
mais do que estudos epidemiológicos para subsidiarem a atividade de gerência de serviços de saúde, os métodos de análise de dados desenvolvidos pela epidemiologia podem ser valiosos instrumentos gerenciais ${ }^{1}$. $O$ compromisso da epidemiologia com a administração dos serviços de saúde tem aumentado nos últimos anos, com crescente literatura sendo produzida, num movimento que tende a, talvez, identificar uma nova disciplina na Saúde Pública ${ }^{2}$.

As diferentes técnicas de análise multivariada constituem poderosos instrumentos de investigação para o campo da Saúde Pública, por permitirem uma apreensão mais abrangente da realidade, excedendo os níveis tradicionais da relação de causa e efeito, ou mesmo de risco controlado por potenciais variáveis de confusão. Esse tipo de investigação tem o mérito de, ao trabalhar informaçốes quantitativas, permitir inferências qualitativas mais genéricas, que sáo de todo pertinentes para um objeto complexo como o planejamento e a gestão do sistema ou dos serviços de saúde.

Curi, em 1993, produziu um estudo que, aplicando técnicas de análise multivariada, permitiu o agrupamento de países segundo indicadores de padrão de vida, extraídos de publicações anuais da Organização das Naçōes Unidas e do Banco Mundial ${ }^{3}$. A inclusão de indicadores sociais, econômicos, demográficos, de saúde, culturais e educacionais dependeu da disponibilidade de informação para todos os 125 países estudados. Comenta o autor que "estas informações na maioria das vezes, têm sido utilizadas sem um enfoque comparativo abrangente $\mathrm{e}$ acabam servindo como elementos ilustrativos de reportagens publicadas em revistas não científicas". Diferentes sistemas de informação social acumulam uma larga gama de dados que, se devidamente processados, podem gerar conhecimento efetivo da realidade, com a vantagem de poupar o investigador do exaustivo e dispendioso processo de coleta de dados originais.

Hahn e colaboradores utilizaram, para obtenção de agregados homogêneos de sub- regióes do Estado de São Paulo, a técnica de análise de agrupamento (Cluster Analysis) e, para medir a eficácia dos grupos obtidos, a técnica de análise discriminatória, aplicadas no conjunto de indicadores extraídos ou calculados a partir de fontes diversas de dados secundários ${ }^{4}$.

No presente trabalho buscou-se exercitar a aplicação dessas técnicas na caracterização de municípios - unidades fundamentais do Sistema Único de Saúde - argüindo-se a hipótese de que a dadas condições sócioeconômicas correspondem situaçôes sanitárias específicas, cujas caracterizaçôes devem auxiliar tanto o diagnóstico de situação quanto a definição de estratégias de intervenção.

\section{Material e métodos}

Tomou-se para estudo o conjunto de municípios do Estado de São Paulo com população igual ou superior a $100 \mathrm{mil}$ habitantes em 1991. Identificaram-se assim 49 municípios, 17 dos quais pertencentes à Região Metropolitana de São Paulo. Como fonte de dados demográficos e sócio-econômicos recorreu-se aos registros do IBGE do ano de 1991. Foram utilizados os seguintes indicadores: renda média dos chefes de domicílios, em salários mínimos (RSM); porcentagem de chefes de domicílios que recebem até 2 salários mínimos (POB); número médio de habitantes por domicílio (HDO); número médio de cômodos por domicílio (COM); porcentagem de domicílios que têm até 3 cômodos (DOM); porcentagem de domicílios com abastecimento de água inadequado (AIN); porcentagem de domicílios com sistema de esgoto inadequado (EIN); porcentagem de domicílios com coleta inadequada de lixo (LIN); porcentagem de pessoas com 15 anos ou mais analfabetas (ANA) e porcentagem de crianças que vivem em domicílios cujos chefes têm menos de 1 ano de escolaridade (CRA). Dos registros da Fundação SEADE foram utilizados: porcentagem de crianças matriculadas em préescolas, públicas ou privadas, em relação ao 
total de crianças. na faixa etária de 4 a 6 anos (MPE); taxa de evasão escolar no ensino público de 1 ㅇ grau - municipal ou estadual (EVA); taxa de reprovação no ensino público de 1 ㅇ grau (REP); relaçáo entre o total de matriculados no $2^{\circ}$ grau sobre o total de matriculados no $1^{\circ}$ grau (SPG) e número de leitos gerais para cada mil habitantes (LEI). Como indicadores de saúde recorreu-se às informações sobre mortalidade para 1992, fornecidos pela Fundação SEADE.

Indicadores de natureza sócioeconômica foram transformados para uma escala uniforme, de forma a permitir a comparação entre os diferentes municípios segundo uma mesma base de mensuração. Por meio dessa metodologia, cada unidade de análise recebeu uma "nota" para cada indicador, variando de 0 a 1 , cabendo a "nota" 0 para o município com o pior indicador e a "nota" 1 para o município com o melhor indicador. A Tabela I mostra os valores máximo (nota 1 ) e mínimo (nota 0) dos indicadores sócioeconômicos originais e os municípios correspondentes.

Coeficientes de mortalidade por causa específica de 1992 foram padronizados segundo a pirâmide etária do Estado de São Paulo em 1991 e reagrupados na forma recomendada pela Organização Pan-Americana de Saúde denominada "6/61"5,6. Essa classificação, compatível com a Classificação Internacional de Doenças, inclui 6 grandes agrupamentos:

- coeficiente padronizado de mortalidade por 10.000 habitantes por doenças infecciosas;

- neoplasias-tumores (TUM);

- doenças do aparelho circulatório;

- causas perinatais (NAT);

- causas extremas e

- todas as outras causas de morte (OUT).

$O$ documento original recomenda o desdobramento, se necessário, dos grupos citados. Assim sendo, o coeficiente de mortalidade por doenças infecciosas foi desdobrado em três subgrupos: enterites (ENT), pneumonias (PNE) e as demais infecciosas (INF); as doenças do sistema circulatório foram desdobradas em : doenças isquêmicas do coração (CORA), as doenças cerebrovasculares (CERE) e as demais do sistema circulatório (CIR); as por causas violentas foram desdobradas em: acidentes de veículos (ACI), os homicídios (HOMI) e as demais causas violentas (VIO). Portanto, este estudo incluiu 12 grupos de causas de óbitos.

Para o reconhecimento de grupos homogêneos de municípios aplicou-se a técnica de análise de agrupamento (Chuster analysis) através das variáveis criadas pela transformação dos indicadores sócio-econômicos. Esta técnica permite o cálculo de distâncias multidimensionais entre os objetos examinados ${ }^{7}$; no caso presente, os municípios. Aplicou-se o método de aproximação centróide utilizandose distâncias Euclidianas. Dispensou-se a padronização das medidas em vista da transformação anterior dos indicadores para uma escala comum. Neste método, observaçóes próximas são agrupadas e novas observaçóes individuais ou novos aglomerados são ajuntados segundo análise das distâncias entre os centros geométricos dos grupos (distância interna média ou centróide de grupo).

Examinou-se, em seguida, a capacidade discriminatória que as condiçóes de saúde teriam para os grupos de municípios identificados. Aplicou-se a técnica de análise discriminante multivariada, tomando-se o agrupamento de municípios segundo condiçóes sócio-econômicas como variável dependente $\mathrm{e}$ os coeficientes de mortalidade por causa específica como variáveis independentes, ou preditoras de grupo. Desta forma, pôde-se aferir se o agrupamento derivado de variáveis sócio-econômicas adequava-se também aos perfis de situação de saúde, conforme indicados pelos perfis de mortalidade. A análise discriminante multivariada ${ }^{7}$, uma técnica semelhante à regressão linear múltipla, dela se diferencia por considerar como dependente uma variável categórica e por derivar a combinação linear entre variável dependente e independentes através da correlação canônica 
destas últimas com várias funçōes complementares geradas pela análise. A análise discriminante multivariada deriva os coeficientes das funçôes discriminantes, buscando maximizar a relação variação entregrupos e variação intra-grupo, enquanto que a regressão linear múltipla gera uma única função, através da técnica dos mínimos quadrados, que busca minimizar os resíduos entre valores preditos e observados.

Para qualificar os grupos identificados quanto a situação sócio-econômica e a situação de saúde, examinou-se as diferenças de média de cada variável por cada grupo através da técnica de análise de variância (ANOVA). Variáveis que num grupo registraram médias estatisticamente diferentes das de outros grupos prestaram-se à qualificação do grupo. A ANOVA, comparando variâncias intra $\mathrm{e}$ intergrupos, permite inferir a probabilidade de dois ou mais grupos serem efetivamente distintos ${ }^{8}$.

Todo processamento estatístico foi feito com auxílio do pacote SPSS-PC (Statistical Package for Social Sciences).

\section{Resultados}

O Quadro I apresenta o dendrograma de agrupamento sucessivo dos municípios que, cotejado com as variaçóes de coeficientes de distância geral a cada estágio de agrupamento, permitiu a identificaçáo de sete grupos distintos de municípios. Estes grupos e seus municípios componentes são apresentados na Tabela II, que registra os valores de cada variável para cada município e a média geral do universo estudado.

O Quadro II mostra o resultado da análise discriminante multivariada onde se conclui que perfis específicos de mortalidade ajustam-se a $83,67 \%$ dos enquadramentos de municípios por grupos derivados das variáveis socioeconômicas. As duas primeiras funçōes derivadas explicam $81,20 \%$ da variação do modelo matemático, destacando-se a contribuiçāo das seguintes variáveis, pela ordem de importância: HOMI (homicídios),
VIO (outras causas violentas), CORA (doenças isquêmica do coração) e CIR (outras doenças do sistema circulatório).

A Tabela III apresenta os coeficientes de mortalidade por município agrupados como a Tabela II, com os índices de cada município, as médias por grupo e a média geral do universo estudado.

Analisando-se as diferenças estatisticamente significantes destas variáveis, classificou-se qualitativamente os sete grupos de municípios, conforme mostra o Quadro III, de acordo com o exame da análise de variância (ANOVA). Os resultados da análise de variância são por demais extensos para apresentação, estando disponíveis a interessados.

O termo "destaque" neste trabalho foi usado para designar índices do grupo significativamente diferentes da maioria dos outros grupos; "predomínio" foi usado para designar índices do grupo significativamente diferentes de todos os outros grupos. Nestas diferenças, "destaque positivo" designa os indicadores sócio-econômicos com médias altas e os índices baixos de mortalidade; "destaque negativo" designa os indicadores sócioeconômicos com médias baixas e os índices altos de mortalidade; "predomínio positivo" aponta os indicadores sócio-econômicos com médias maiores que todos os outros grupos e os indicadores de saúde com menores índices de mortalidade; "predomínio negativo" aponta os indicadores sócio-econômicos com médias menores que todos os outros grupos e os piores índices de mortalidade.

Os municípios divergentes (outliers) apontados pela análise discriminante estão relacionados na Tabela IV. Este quadro mostra o grupo observado, ao qual pertence o município segundo a análise de agrupamento c o grupo predito, indicado pela análise discriminante, junto com as respectivas probabilidades de pertencer a um ou outro grupo. 


\section{Comentários e conclusões}

Este trabalho demonstra a potencialidade do uso de dados secundários e a aplicação de técnicas de análise multivariada no reconhecimento da complexa realidade social e sanitária que solicita um projeto de intervenção. A escolha de indicadores foi influenciada pela disponibilidade de dados secundários. Uma situação concreta deverá cotejar essa disponibilidade de informação com as necessidades do sistema de saúde ou metas de planejamento do administrador de saúde. A possibilidade de discernir grupos homogêneos de municípios segundo uma extensa gama de fatores de diferentes aspectos da realidade sócio-econômica e de saúde pode auxiliar a categorização desta realidade e sugerir estratégias comuns de intervenção.

A identificação de cenários sócioeconômicos com perfís sanitários correspondentes não estabelece relação de causa e efeito entre as duas situaçōes, de forma que não se pode inferir que alteraçōes de uma impliquem em variaçóes da outra. No entanto, esta caracterização simultânea das condições sócio-econômicas e de saúde sugere, de forma consistente, os itens de ambas as extraçóes que devem ser objeto de prioridade num projeto de intervenção. Algumas das variáveis podem contemplar projetos integrados de controle sem que se passe pela preocupação de sua eventual relação de causa e efeito, mas simplesmente pela consideração de um diagnóstico conjuntural.

O grupo 7 do Quadro III tem, simultaneamente, problemas de enterite como causa de óbito e problemas de lixo e esgoto como problemas de infra-estrutura social: independentemente de qualquer demonstraçáo de relação de causa e efeito entre os eventos, eles podem contemplar programas integrados de controle para uma abordagem simultânea.

O mesmo Quadro III pode ser observado de outra maneira. Os grupos definidos pela análise de agrupamento recebem denominação numérica, qualificando-os de grupo 1 (com maior número de indicadores sócio-econômicos em destaque positivo) até grupo 7 (com maior número desses indicadores em destaque negativo). Já a diferença de indicadores de saúde entre os grupos não reconhece ordem de hierarquia, mas apenas categorização qualitativa. Da mesma forma que se presta à distinção dos grupos, a qualificação por indicadores de saúde permite igualmente a identificação de novas formas de agrupamento. De fato, considerando-se as variáveis mais destacadas na análise discriminante (óbitos por homicídios e óbitos por outras causas violentas), os grupos de municípios poderiam ajuntar-se em dois supergrupos: os grupos $1,2,3$ e 4 que se caracterizam por baixos índices de óbitos por homicídios e por outras causas violentas (SUPERGRUPO A) e os grupos 5,6 e 7, cuja característica é a presença significativamente elevada dos mesmos índices (SUPERGRUPO B). Esses supergrupos, assim identificados, mostram homogeneidade também em relaçãu aos indicadores sócio-econômicos, uma vez que a passagem do grupo 4 para o grupo 5 é marcada pela inversão dos destaques positivos para os destaques negativos.

A aplicação dos métodos analíticos multivariados permite recuperar a avaliação qualitativa do nível discursivo para o nível operacional. Menos preocupados com a precisão das medidas que realizam, esses métodos permitem, como demonstrado neste exercício, caracterizaçóes qualitativas que se expressam como destaque e predominância de diferenças. Ao contrário das avaliaçōes exclusivamente quantitativas, análises desse tipo permitem que mesmo as eventuais inconsistências do modelo contribuam para uma reflexão e conclusão produtivas.

De fato, a análise discriminante multivariada apresentada no Quadro II, ao apontar a concordância entre valores preditos e observados ao nível de $83,67 \%$ ao invés de sugerir limites do modelo de análise, permite a identificação de casos divergentes e a 
interpretação dos fatores de desvio para classificação da observação. Na Tabela III, estes casos divergentes que respondem pelo resíduo de discordância do modelo $(16,33 \%)$ são identificados e seu desvio pode ser interpretado. Bauru e Rio Claro sáo classificados erroneamente pelo modelo discriminante, destoando do grupo original principalmente nos índices de mortalidade por doenças cerebrovasculares. Rio Claro, assim como São Carlos, apontam valores mais altos do que o grupo original no índice de mortalidade por tumores. Note-se que os índices de mortalidade por homicídios e por doenças isquêmicas do coraçáo têm um peso grande na correção da classificação. Esta forma permite que o planejador em saúde identifique itens específicos na elaboração de planos de atenção à saúde.

A Tabela IV mostra que, dos oito municípios de classificaçóes divergentes (observada e predita), apenas um é apontado como de supergrupo diferente. $\mathrm{O}$ município de Sumaré apresenta todos os índices de mortalidade menores que as médias de seu grupo. Pertence ao grupo 5 (SUPERGRUPO B) de acordo com indicadores sócioeconômicos e ao grupo 2 (SUPERGRUPO A) pelos indicadores de saúde. Esse mesmo quadro aponta que o município de São Carlos tem probabilidades A e $\mathrm{D}$ muito próximas, isto é, seu perfil de saúde pode encaixar-se tanto no grupo 2 (grupo observado) quanto no grupo 4 (grupo predito). Isso não acontece com os municípios de Rio Claro e Santa Bárbara D'Oeste, cujas probabilidades de pertencerem ao grupo observado sequer aparecem na análise discriminante.

Este estudo incluiu 17 municípios da Região Metropolitana de São Paulo, 10 dos quais concentram-se no grupo 5 , mostrando uma relativa homogeneidade nas características dessas cidades que circundam a Capital.
Do presente trabalho conclui-se que os métodos analíticos multivariados de uso corrente na epidemiologia moderna, se transferidos com criatividade para o campo da Administração em Saúde Pública, poderão trazer importante contribuição, tanto ao diagnóstico de saúde quanto às definiçóes de políticas e programas.

\section{Agradecimentos}

Ao Pólis - Instituto de Estudos, Formação e Assessoria em Políticas Sociais, ONG de São Paulo, pelo acesso ao Banco de Dados de Indicadores Municipais.

\section{Bibliografia}

1. Vaughan, J.P.; Morrow, R.H. Manual of epidemiology for district health management. Geneva: World Health Organization: vi, 1989.

2. Woodward, M.; Francis, L. M. A. Statistics for health management and research. London: Edward Arnold: 2-7, 1988.

3. Curi, P. R. Agrupamento de países segundo indicadores de padrão de vida. Revista de Saúde Pública, 27(2):127-34, 1993.

4. Hahn, N. S.; Bussab, W. O.; Ho L. L. Características Regionais de Saúde no Estado de São Paulo - FUNDAP - Dez. 1983.

5. Castellanos, P. L. Health situation analysis and inequities in health - Working paper PAHO/WHO, Nov. 1992.

6. Organizacion Panamericana de la Salud. El análisis de la situación de salud: base de la gestión en salud y de la cooperación técnica - Reunión de Gerentes 1992 Washington, diciembre 1992.

7. Hair, J. F.; Anderson, R. E.; Tatham, R. L. Multivariate data analysis. New York: MacMillan Publishing Co.: 73-347, 1987.

8 Armitage, P.; Berry, G. Statistical methods in medical research. London: Blackwell Scientific Publications: 186:93, 1987. 
Tabela I - Valores máximo e mínimo originais dos indicadores sócio-econômicos usados na análise de agrupamento e seus respectivos municípios transformados em notas 1 e 0

\begin{tabular}{|c|c|c|}
\hline INDICADORES & $\begin{array}{l}\text { MUNICÍPIOS } \\
\text { NOTA } 1 \\
\end{array}$ & $\begin{array}{l}\text { MUNICÍPIOS } \\
\text { NOTA } 0 \\
\end{array}$ \\
\hline $\begin{array}{l}\text { renda média em salários mínimos } \\
\text { (RSM) }\end{array}$ & 7,2 (São Paulo) & 2,8 (Itaquaquecetuba) \\
\hline $\begin{array}{l}\text { \% chefes que ganham até } 2 \\
\text { salários mínimos (POB) }\end{array}$ & 23,7 (Santos) & 47,3 (Itapetininga) \\
\hline $\begin{array}{l}\text { número de habitantes por } \\
\text { domicílio (HDO) }\end{array}$ & 3,45 (S antos) & 4,36 (Itaquaquecetuba) \\
\hline $\begin{array}{l}\text { número de cômodos por } \\
\text { domicílio }(C O M)\end{array}$ & 6,13 (Araçatuba) & 3,96 (Itaquaquecetuba) \\
\hline $\begin{array}{l}\text { \% domicílios com } 3 \text { cômodos } \\
\text { (DOM) }\end{array}$ & 9,3 (Mogiguaçu) & 45,3 (Diadema) \\
\hline $\begin{array}{l}\text { \% domicílios com água } \\
\text { in adequada ( } \mathrm{AIN} \text { ) }\end{array}$ & 0,3 (São Caetano) & 11,2 (Itaquaquecetuba) \\
\hline $\begin{array}{l}\% \text { domicílios com esgoto } \\
\text { inadequado (EIN) }\end{array}$ & 0,4 (São Caetano) & 67,6 (Sumaré) \\
\hline $\begin{array}{l}\text { \% domicílios com coleta de lixo } \\
\text { in adequada (IIA) }\end{array}$ & 0,1 (São Caetano) & 22,5 (Itapevi) \\
\hline $\begin{array}{l}\% \text { de analfabetos com } 15 \text { anos ou } \\
\text { mais (ANA) }\end{array}$ & 5,4 (S antos) & 14,5 (Itaquaquecetuba) \\
\hline $\begin{array}{l}\text { \% crianças com chefe de família } \\
\text { analfabeto (CRA) }\end{array}$ & 5,9 (São Caetano) & 19,9 (Guarujá) \\
\hline $\begin{array}{l}\text { \% crianças matriculadas na pré- } \\
\text { escola (MPE) }\end{array}$ & 88,9 (M arília) & 2,2 (Itapevi) \\
\hline $\begin{array}{l}\text { taxa de evasão } 1^{\circ} \text { grau - escolas } \\
\text { públicas (EVA) }\end{array}$ & 6,8 (São Bernardo) & 13,0 (Presidente Prudente) \\
\hline $\begin{array}{l}\text { taxa de reprovação } 1^{\circ} \text { grau - } \\
\text { escolas públicas (REP) }\end{array}$ & 8,0 (Araçatuba) & 21,4 (Guarujá) \\
\hline $\begin{array}{l}\text { relação matriculados } 2^{\circ} \text { sobre } 1^{\circ} \\
\text { grau (SPG) }\end{array}$ & 36,4 (São Caetano) & $6,2(\mathrm{Embu})$ \\
\hline $\begin{array}{l}\text { número de leitos gerais por mil } \\
\text { habitantes (LEI) }\end{array}$ & 8,7 (Jundiaí) & 0 (Embu, Itaquaquecetuba) \\
\hline
\end{tabular}

Fontes: IBOE • Fundaçä̊ SEADE - 1991 


\section{Quadro I - Dendograma do agrupamento de municíplos}

Dendrograma usando aproximação média entre os grupos

Distâncias dos agrupamentos

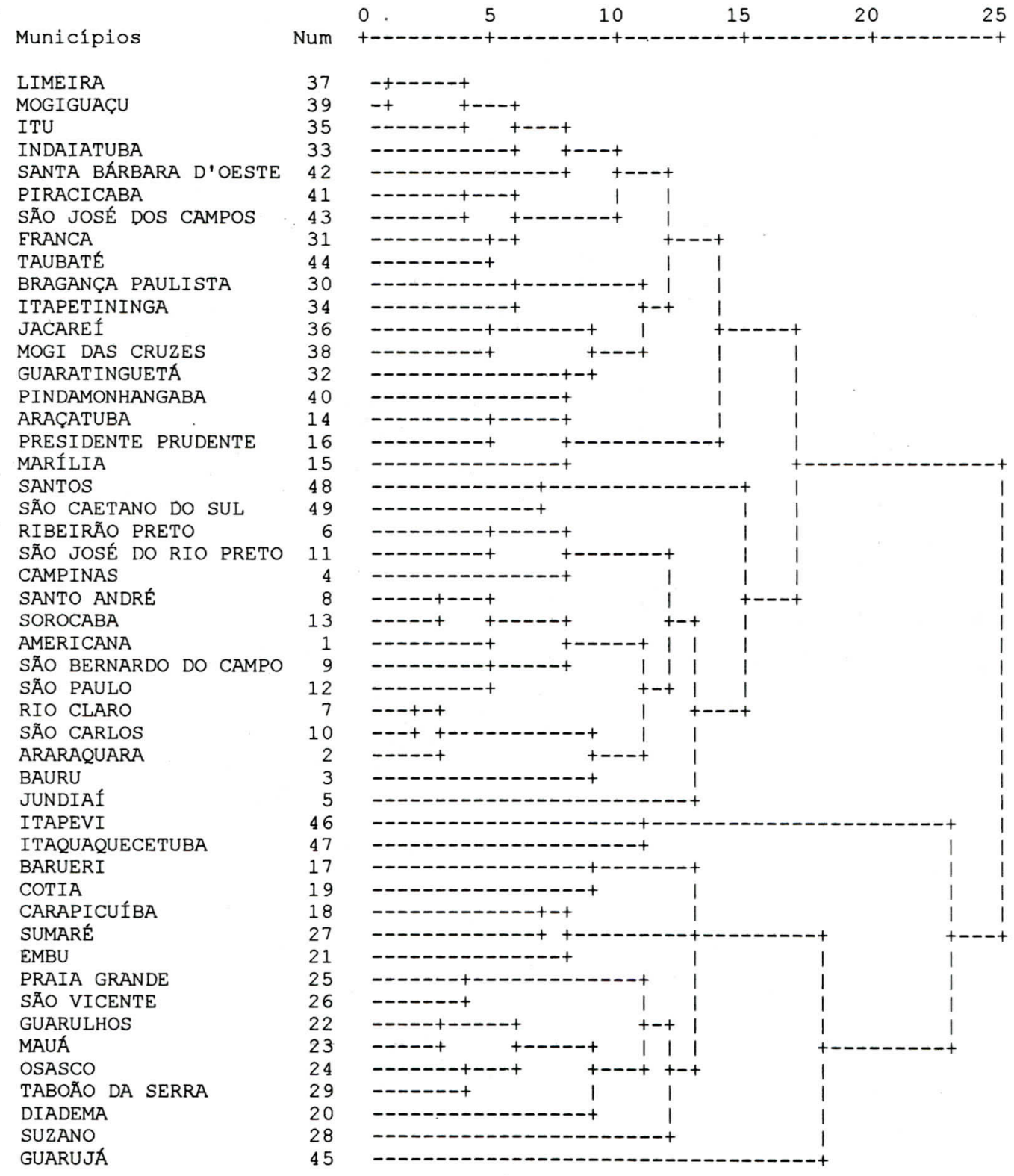


Mortalidade em Municípios de SP e Indicadores Sócio-económicos: uma Análise de Agrupamento

Tabela II - Relação dos municíplos estudados, agrupados segundo seus indicadores sócio-econômicos

\begin{tabular}{|c|c|c|c|c|c|c|c|c|c|c|c|c|c|c|c|}
\hline & RSM & POB & HIDO & $\infty \mathrm{M}$ & DaM & AIN & EIN & LN & ANA & CRA & MPE & EVA & REP & SPG & IEI \\
\hline 1 SANTOS &, 955 & 1,00 & 1,00 &, 926 &, 972 &, 881 &, 894 & ,960 & 1,00 & ,814 &, 533 &, 581 &, 590 &, 788 & ,621 \\
\hline S.CAETANOSUL & 977 & ,941 & ,989 &, 756 &, 828 & 1,00 & 1,00 & 1,00 &, 978 & 1,00 &, 904 &, 823 &, 709 & 1,00 &, 575 \\
\hline 2 AMERICANA &, 591 & ,788 & ,582 &, $7 \pi 0$ & ,828 & ,835 & ,914 & 879 &, 758 & ,943 & ,619 & ,935 & ,612 &, 563 &, 529 \\
\hline ARARAQUARA &, 500 & 631 & 637 &, 903 &, 950 &, 945 & 949 &, 844 &, 637 &, 729 &, 752 & 629 & ,970 & ,490 & ,483 \\
\hline BAURU &, 568 &, 602 & ,637 & 1,00 &, 997 &, 743 & ,859 & ,384 &, 714 &, 750 &, 660 &, 532 &, 597 &, 500 & ,368 \\
\hline CAMPINAS & 97 & 941 & 659 &, 894 & ,861 &, 706 & 799 &, 866 &, 758 &, 750 & ,270 & ,581 &, 425 &, 474 &, 425 \\
\hline JUNDIAI & ,614 &, 636 &, 549 & ,608 &, 736 &, 927 &, 857 &, 924 & 692 & ,764 & ,392 & ,710 & ,388 & ,414 & 1,00 \\
\hline RIBEIRĀOPREIO &, 795 &, 864 & 571 & ,991 & 972 &, 908 & ,935 & 879 & ,857 & ,914 & 392 & 274 &, 642 & 464 &, 494 \\
\hline RIOCLARO & ,409 &, 525 & ,637 &, 866 & ,983 & ,945 & ,952 &, 920 &, 736 &, 693 & ,842 &, 742 &, 679 &, 457 & 276 \\
\hline SANIO ANDRÉ &, 682 & 831 &, 626 &, 567 &, 650 & 844 & ,905 &, 978 & 868 & 843 & ,582 &, 726 &, 515 &, 599 & 264 \\
\hline S.BERNARDOCAMPO &, 932 & 970 &, 527 &, 567 & ,625 &, $6 \pi 0$ &, 795 & ,933 &, 813 &, 714 &, 731 & 1,00 &, 567 &, 430 & 253 \\
\hline SĀOCARLOS & ,455 &, 725 & ,538 &, 926 &, 972 &, 927 & ,958 & ,888 &, 582 &, 743 &, 669 &, 887 &, 784 &, 540 & ,310 \\
\hline S.JOSÉ RIO PREIO &, 591 & 695 & , 791 & ,968 & ,961 & ,954 & ,937 & ,951 & $6 \pi 0$ &, 900 &, 597 & 290 & ,425 & ,483 &, 701 \\
\hline SÄOPAULO & 1,00 & ,856 & 670 & ,608 &, 572 & ,853 &, 853 & ,951 &, 769 & 607 & , 463 &, 661 &, 590 &, 424 &, 345 \\
\hline SOROCABA &, 545 & 640 & 429 & ,599 &, 708 & ,881 & ,946 &, 902 &, 802 & 829 &, 607 &, 565 & 619 &, 450 & ,379 \\
\hline 3 ARAÇATUBA & ,386 & , 157 &, 577 & 1,00 & 969 &, 725 &, 893 &, 877 & ,538 &, 643 & ,656 & ,274 & 1,00 &, 589 &, 322 \\
\hline MARTIIA &, 409 & 242 &, 440 &, 986 & ,956 &, 624 &, 887 &, 790 & ,418 &, 643 & 1,00 & 194 & 813 &, 556 & 747 \\
\hline PRESID. PRUDENIE & 477 & 275 &, 593 & ,968 & ,914 &, 624 &, 707 &, 732 & ,473 &, 736 & 461 & 0,00 &, 784 & ,639 & ,494 \\
\hline 4 BRAGANÇA PAULISTA &, 364 & 119 & ,484 &, 664 & ,808 &, 817 &, 705 &, 737 & 187 & ,529 & ,488 & ,161 &, 687 & ,361 & ,460 \\
\hline FRANCA & ,341 &, 513 & ,451 &, 783 & 872 & ,908 &, 964 & ,955 & ,648 &, 857 &, 434 &, 048 & 649 &, 401 & 264 \\
\hline GUARATINGUETÁ & ,386 & 123 & 297 & 806 & 844 & 651 &, 772 & ,759 &, 736 &, 786 & 396 &, 565 & ,858 &, 464 &, 575 \\
\hline INDAIATUBA & ,455 &, 487 & 396 & ,558 & ,825 &, 927 & 854 &, 929 & ,451 &, 629 &, 452 &, 597 & ,351 & 202 & ,115 \\
\hline ITAPETININGA & 205 & 0,00 & ,385 &, 622 & ,786 & 468 &, 729 &, 705 &, 440 &, 664 &, 584 &, 065 & ,418 & 371 &, 425 \\
\hline IIU & 432 & ,398 & 363 & 682 & ,803 &, 972 & ,905 & 862 & ,352 &, 464 &, 571 & 629 &, 567 & 291 &, 483 \\
\hline JACAREI & ,341 & ,309 & 341 & ,484 &,$\pi 06$ &, 670 & ,649 &, 830 & ,582 &, 664 & ,348 &, 565 & 634 &, 427 & 241 \\
\hline LIMRIRA & 295 &, 364 & ,418 & ,733 &, 894 & ,936 & ,985 & ,942 & ,538 &, 736 &, 565 &, 500 & ,590 & ,434 &, 368 \\
\hline MOGI DAS CRUZES &, 455 & 390 & 286 &, 470 & ,625 & ,330 &, 725 &, 750 &, 527 &, 493 & ,436 &, 581 &, 493 &, 513 & ,494 \\
\hline MOGIGUAÇU &, 250 &, 343 & ,319 & ,733 & 1,00 & 890 & ,984 & ,955 & ,374 &, 757 &, 529 &, 565 &, 739 & 348 & 264 \\
\hline PINDAMONHANGABA & 273 & ,110 &, 044 &, 774 & ,917 & $6 \pi 0$ & 644 &, 661 & 604 &, 664 &, 421 & ,355 &, 500 & 288 & 253 \\
\hline PIRACICABA &, 568 &, 691 &, 505 & ,816 &, 947 & 807 & ,915 &, 866 & ,747 &, 757 & ,444 & ,403 &, 545 & ,381 &, 322 \\
\hline S.BÁRBARADOESTE & , 182 & ,487 & 264 &, 548 & 800 & 890 & ,963 &, 902 & ,516 &, 77 & ,315 & ,968 & 619 & 146 & 126 \\
\hline S.JOSÉ CAMPOS &, 682 & 648 & 286 &, 728 & 839 & 615 &, 827 &, 933 &, 813 & ,857 & 37 &, 516 &, 739 & 421 & 448 \\
\hline TAUBATÉ & ,455 & ,347 & 264 &, 793 & 892 & 844 & 812 &, 875 &, 780 & 814 &, 480 & ,387 &, 500 &, 460 & 195 \\
\hline 5 BARUERI &, 773 &, 525 & , 154 &, 364 & ,181 & ,459 &, 499 &, 875 & ,352 & 293 &, 732 & ,371 &, 410 & 166 &, 023 \\
\hline CARAPICUIBA &, 227 &, 593 & 176 & 189 &, 350 & ,596 & 180 & 879 & ,462 &, 464 &, 022 & ,387 &, 567 & 123 &, 046 \\
\hline COIA &, 705 &, 572 & 165 &, 507 & ,422 &, 642 & , 132 &, 549 &, 396 & ,407 &, 408 & ,435 &, 343 & 179 & 207 \\
\hline DIADEMA &, 227 & ,597 &, 363 & ,018 & 0,00 & ,761 &, 680 & ,933 & 198 & 264 &, 464 & 613 & ,433 & 159 & ,264 \\
\hline EMBU & 182 &, 530 &, 088 & 138 & 244 &, 725 &, 278 & ,848 &, 099 &, 214 & ,188 & ,339 & 276 & 0,00 & 0,00 \\
\hline GUARUIHOS &, 409 & 648 & ,341 & 249 & 325 &, 505 & ,467 & ,665 & ,462 & ,464 & 230 &, 726 & ,433 & ,252 & 241 \\
\hline MAUÁ &, 250 &, 619 &, 275 & 161 & 264 &, 642 & ,729 &, 728 &, 451 & ד & 291 &, 500 & ,388 & ,225 & ,218 \\
\hline OSASCO &, 432 & 699 &, 418 &, 304 & ,358 &, 817 & ,632 & 946 &, 604 &, 614 &, 329 & ,468 & 649 & 371 & 207 \\
\hline PRAIAGRANDE &, 227 & 318 & $6 \pi 0$ &, 323 &, 594 &, 560 &, 350 & ,746 & 462 &, 450 &, 487 &, 742 & 239 & 136 &, 092 \\
\hline SÃO VICENIE &, 318 &, 538 &, 560 &, 382 &, 717 & ,716 &, 537 &, 625 &, 626 &, 550 &, 368 &, 774 & 284 & 242 & 161 \\
\hline SUMARÉ & 136 & 419 & 132 &, 378 & 639 &, 578 & 0,00 & ,768 & 198 &, 464 & 298 & ,468 & ,433 & 149 &, 069 \\
\hline SUZANO & 250 & 297 & 143 &, 323 & ,478 &, 404 &, 440 &, 545 & 330 &, 421 & 279 &, 726 &, 724 & 238 & ,391 \\
\hline TABOĀODA SERRA & 409 &, 725 & 286 & ,341 & ,428 &, 752 &, 658 & ,969 &, 473 &, 464 & 379 &, 548 &, 373 &, 175 &, 034 \\
\hline 6 GUARUJA &, 114 &, 076 & ,308 & ,300 & ,531 & ,358 &, 774 & ,799 &, 099 & 0,00 & 260 &, 645 & 0,00 &, 156 & 276 \\
\hline 7 ITAPEVI &, 091 & 123 & ,011 & , 161 &, 256 &, 064 & 268 & 0,00 & ,242 & ,357 & 0,00 & 161 &, 560 &, 043 & ,011 \\
\hline ITAQUAQUECETUBA & 0,00 &, 042 & 0,00 & 0,00 &, 069 & 0,00 & 079 &, 500 & 0,00 & 179 &, 082 & 484 & 276 &, 013 & 0,00 \\
\hline
\end{tabular}




\section{Quadro II - Resultados da análise discriminante}

Parte 1 - As funções discriminantes

Funções Canônicas

Função \% razão de Variância \% variância entre/inter

: Correlaçẫo grupos
Funções Discriminantes

Função Wilks' Qui-

Lambda quadrado

\begin{tabular}{|c|c|c|c|c|c|c|c|c|c|}
\hline & & & & & 0 & 0135797 & 165,51849 & 72 & ,0000 \\
\hline $1^{*}$ & 4,85640 & 54,31 & 54,31 & ,9106297 & 1 & ,0795279 & 97,46842 & 55 & 0004 \\
\hline $2^{*}$ & 2,40427 & 26,89 & 81,20 & ,8403878 & 2 & 2707349, & 50,30469 & 40 & , 1274 \\
\hline $3^{*}$ & ,94651 & 10,58 & 91,78 & 6973232 & 3 &, 5269878 & 24,66225 & 27 & ,5934 \\
\hline $4^{*}$ & ,40128 & 4,49 & 96,27 &, 5351347 & 4 & ,7384599 & 11,67275 & 16 & ,7662 \\
\hline $5^{*}$ & 25193 & 2,82 & 99,09 & 4485909, & 5 & ,9245008 & 3,02230 & -7 & ,8829 \\
\hline $6^{*}$ & 08166 & ,91 & 100,00 & 2747712 & & & & & \\
\hline
\end{tabular}

- Marca as 6 funçōes canônicas discriminantes extraídas na análise.

Parte 2 - Tabela de concordância entre os grupos formados pela análise de agrupamento (mortalidade) e os grupos formados pela análise discriminante

\begin{tabular}{|c|c|c|c|c|c|c|c|c|}
\hline \multirow{2}{*}{$\begin{array}{l}\text { Análise } \\
\text { Agrupamento }\end{array}$} & \multirow{2}{*}{$\begin{array}{l}\text { № de } \\
\text { Casos }\end{array}$} & \multirow[b]{2}{*}{1} & \multicolumn{6}{|c|}{ Análise Discriminante } \\
\hline & & & 2 & 3 & 4 & 5 & 6 & 7 \\
\hline Grupo 1 & 2 & $\begin{array}{c}2 \\
100,0 \%\end{array}$ & $\begin{array}{c}0 \\
, 0 \%\end{array}$ & $\begin{array}{c}0 \\
, 0 \%\end{array}$ & $\begin{array}{c}0 \\
, 0 \%\end{array}$ & $\begin{array}{c}0 \\
, 0 \%\end{array}$ & $\begin{array}{c}0 \\
, 0 \%\end{array}$ & $\begin{array}{c}0 \\
, 0 \%\end{array}$ \\
\hline Grupo 2 & 13 & $\begin{array}{c}0 \\
, 0 \%\end{array}$ & $\begin{array}{c}10 \\
76,9 \%\end{array}$ & $\begin{array}{c}2 \\
15,4 \%\end{array}$ & $\begin{array}{c}1 \\
7,7 \%\end{array}$ & $\begin{array}{c}0 \\
, 0 \%\end{array}$ & $\begin{array}{c}0 \\
, 0 \%\end{array}$ & $\begin{array}{c}0 \\
, 0 \%\end{array}$ \\
\hline Grupo 3 & 3 & $\begin{array}{c}0 \\
, 0 \%\end{array}$ & $\begin{array}{c}0 \\
, 0 \%\end{array}$ & $\begin{array}{c}3 \\
100,0 \%\end{array}$ & $\begin{array}{c}0 \\
, 0 \%\end{array}$ & $\begin{array}{c}0 \\
, 0 \%\end{array}$ & $\begin{array}{c}0 \\
, 0 \%\end{array}$ & $\begin{array}{c}0 \\
, 0 \%\end{array}$ \\
\hline Grupo 4 & 15 & $\begin{array}{c}0 \\
, 0 \%\end{array}$ & $\begin{array}{c}3 \\
20,0 \%\end{array}$ & $\frac{1}{6,7 \%}$ & $\begin{array}{c}11 \\
73,3 \%\end{array}$ & $\begin{array}{c}0 \\
, 0 \%\end{array}$ & $\begin{array}{c}0 \\
, 0 \%\end{array}$ & $\begin{array}{c}0 \\
, 0 \%\end{array}$ \\
\hline Grupo 5 & 13 & $\begin{array}{c}0 \\
, 0 \%\end{array}$ & $\frac{1}{7,7 \%}$ & $\begin{array}{c}0 \\
, 0 \%\end{array}$ & $\begin{array}{c}0 \\
, 0 \%\end{array}$ & $\begin{array}{c}12 \\
92,3 \%\end{array}$ & $\begin{array}{c}0 \\
, 0 \%\end{array}$ & $\begin{array}{c}0 \\
, 0 \%\end{array}$ \\
\hline Grupo 6 & 1 & $\begin{array}{c}0 \\
, 0 \%\end{array}$ & $\begin{array}{c}0 \\
, 0 \%\end{array}$ & $\begin{array}{c}0 \\
, 0 \%\end{array}$ & $\begin{array}{c}0 \\
, 0 \%\end{array}$ & $\begin{array}{c}0 \\
, 0 \%\end{array}$ & $\begin{array}{c}1 \\
100,0 \%\end{array}$ & $\begin{array}{c}0 \\
, 0 \%\end{array}$ \\
\hline Grupo 7 & 2 & $\begin{array}{c}0 \\
, 0 \% \\
\end{array}$ & $\begin{array}{c}0 \\
, 0 \% \\
\end{array}$ & $\begin{array}{c}0 \\
, 0 \% \\
\end{array}$ & $\begin{array}{c}0 \\
, 0 \% \\
\end{array}$ & $\begin{array}{c}0 \\
, 0 \% \\
\end{array}$ & $\begin{array}{c}0 \\
, 0 \% \\
\end{array}$ & $\begin{array}{c}2 \\
100,0 \%\end{array}$ \\
\hline
\end{tabular}

Porcentagem de casos corretamente classificados: $83,67 \%$ 
Mortalidade em Municípios de SP e Indicadores Sócio-econômicos: uma Análise de Agrupamento

Tabela III - Coeficientes padronizados de mortalidade ${ }^{\star *}$ dos municípios estudados

\begin{tabular}{|c|c|c|c|c|c|c|c|c|c|c|c|c|}
\hline & INF & ENT & PNE & TUM & CIR & CORA & CERE & NAT & VIO & ACI & $\begin{array}{r}\mathrm{HOM} \\
\mathrm{I} \\
\end{array}$ & OUT \\
\hline I SANTOS & 2,60 & ,44 & 3,71 & 11,67 & 5,60 & 7,65 & 6,04 & 3,09 & 3,30 & 1,82 & 1,38 & 23,32 \\
\hline S.CAETANO DO SUL & 1,67 &, 22 & 2,60 & 11,84 & 5,83 & 11,99 & 4,53 & 2,46 & 2,06 & 1,52 & 1,98 & 17,22 \\
\hline MÉDIA GRUPO & 2,13 &, 33 & 3,16 & 11,76 & 5,72 & 9,82 & 5,29 & 2,78 & 2,68 & 1,67 & 1,68 & 20,27 \\
\hline 2 AMERICANA &, 87 &, $\mathbf{4 3}$ & 4,97 & 8,74 & 4,67 & 4,88 & 5,39 & 2,74 & 3,76 & 3,05 & 1,28 & 15,23 \\
\hline ARARAQUARA & 1,01 &, 30 & 3,15 & 8,07 & 6,31 & 8,55 & 5,27 & 2,42 & 2,49 & 2,13 &, 92 & 15,15 \\
\hline BAURU * & 1,98 & ,65 & 3,16 & 8,42 & 6,74 & 7,18 & 8,15 & 2,11 & 2,62 & 2,76 &, 78 & 15,76 \\
\hline CAMPINAS & 1,41 & $\mathbf{4 0}$ & 4,89 & 9,29 & 4,59 & 8,12 & 4,68 & 2,22 & 3,59 & 1,86 & 2,32 & 15,71 \\
\hline JUNDIAÍ & 1,60 & ,20 & 4,77 & 10,11 & 5,87 & 7,77 & 6,41 & 2,08 & 4,01 & 1,58 &, 90 & 14,88 \\
\hline RIBEIRĀO PRETO & 2,32 &, 45 & 2,99 & 7,46 & 5,53 & 6,96 & 5,03 & 1,92 & 2,31 & 2,40 & 1,34 & 18,27 \\
\hline RIO CLARO * & 1,65 &, 80 & 3,60 & 9,27 & 6,14 & 5,93 & 8,14 & 2,01 & 2,17 & 2,50 & 1,97 & 15,01 \\
\hline SANTO ANDRÉ & 1,53 &, 40 & 3,74 & 8,56 & 5,90 & 7,15 & 5,31 & 2,72 & 2,63 & 2,31 & 2,55 & 15,39 \\
\hline S.BERNARDO CAMPO & 1,71 & 67 & 3,92 & 8,22 & 6,24 & 8,06 & 6,42 & 3,14 & 2,78 & 2,65 & 3,90 & 14,38 \\
\hline SĀO CARLOS * & 1,67 & ,31 & 3,27 & 9,40 & 5,43 & 5,53 & 6,88 & 2,64 & 2,46 & 1,50 & 1,33 & 15,00 \\
\hline S.JOSÉ RIO PRETO & 3,10 & ,17 & 3,17 & 8,86 & 6,02 & 4,73 & 5,37 & 2,07 & 3,18 & 2,03 & 1,08 & 18,45 \\
\hline SĀO PAULO & 2,05 & ,43 & 4,59 & 9,32 & 5,45 & 7,89 & $\mathbf{5 , 5 7}$ & 2,74 & 2,87 & 1,91 & 3,96 & 16,29 \\
\hline SOROCABA & 1,89 &, 54 & 4,18 & 9,77 & 5,77 & 8,64 & 5,85 & 2,53 & 3,76 & 1,69 & 1,38 & 19,57 \\
\hline MÉDIA GRUPO & 1,75 &, 44 & 3,88 & 8,88 & 5,74 & 7,03 & 6,04 & 2,41 & 2,97 & 2,18 & 1,82 & 16,08 \\
\hline 3 ARAÇATUBA & 1,95 & ,56 & 2,11 & 9,06 & 5,91 & 5,17 & 7,21 & 2,76 & 3,04 & 2,47 & 1,47 & 14,56 \\
\hline MARÍlIA & 2,36 &, 54 & 3,52 & 9,96 & 6,04 & 4,23 & 6,62 & 2,07 & 2,57 & 2,50 &, 71 & 15,65 \\
\hline $\begin{array}{l}\text { PRESID. } \\
\text { PRUDENTE }\end{array}$ & 1,70 & ,61 & 1,97 & 8,97 & 5,84 & 4,64 & 8,30 & 2,82 & 1,72 & 2,19 & 1,88 & 15,80 \\
\hline MÉDIA GRUPO & 2,00 &, 57 & 2,53 & 9,33 & 5,93 & 4,68 & 7,38 & 2,55 & 2,44 & 2,39 & 1,35 & 15,34 \\
\hline $\begin{array}{l}4 \text { BRAGANÇA } \\
\text { PAULISTA }\end{array}$ & 2,00 &, 51 & 3,97 & 10,94 & 7,10 & 5,87 & 8,36 & 4,30 & 2,60 & 4,78 &, 73 & 21,60 \\
\hline FRANCA & 3,57 &, 43 & 4,09 & 8,67 & 7,41 & 4,58 & 6,40 & 2,60 & 3,98 & 2,35 &, 58 & 16,15 \\
\hline GUARATINGUETÁ & 1,20 & ,96 & 2,57 & 8,76 & 10,94 & 7,19 & 6,87 & 2,10 & 3,10 & 1,90 & $1, \mathbf{4 0}$ & 20,09 \\
\hline INDAIATUBA * & 1,41 & ,11 & $\mathbf{4 , 4 3}$ & 8,31 & 6,34 & 8,13 & 6,84 & 2,66 & 3,21 & 1,20 & 1,09 & 11,01 \\
\hline ITAPETININGA & 2,46 & ,70 & 4,20 & 10,60 & 8,47 & 7,39 & 6,00 & 4,14 & 3,21 & 3,62 & 1,26 & 17,66 \\
\hline ITU & 2,14 &, 46 & 3,32 & 9,97 & 7,20 & 7,30 & 7,05 & 3,19 & 2,85 & 2,14 & 1,99 & 17,63 \\
\hline JACAREI & 1,71 &, 44 & 3,20 & 8,65 & 7,03 & 6,30 & 9,27 & 3,15 & 2,43 & 2,84 & 1,58 & 16,25 \\
\hline LIMEIRA & 1,46 &, 37 & 3,11 & 8,78 & 7,89 & 5,24 & 7,94 & 3,24 & 2,13 & 2,96 &, 80 & 16,66 \\
\hline MOGI DAS CRUZES & 1,82 & ,62 & 3,49 & 9,74 & 7,15 & 7,19 & 7,82 & 3,20 & 2,58 & 2,49 & 1,80 & 16,56 \\
\hline MOGIGUAÇU & 1,96 & ,32 & 4,18 & 8,21 & 7,65 & 6,01 & 5,35 & 2,55 & 2,23 & 3,27 &, 41 & 14,32 \\
\hline PINDAMONHANGABA & 1,68 & ,11 & 3,72 & 8,30 & 7,30 & 7,85 & 8,16 & 1,88 & 1,73 & 1,60 & 1,18 & 19,53 \\
\hline PIRACICABA * & 1,96 & ,63 & 3,27 & 9,47 & 6,49 & 6,34 & 5,39 & 2,53 & 2,56 & 1,31 & 2,28 & 17,28 \\
\hline S.BARBARA D'OESTE & 1,54 & ,31 & 2,05 & 8,72 & 5,69 & 4,35 & 5,29 & 1,83 & 3,07 & 1,24 & ,91 & 14,62 \\
\hline S.JOSÉ DOS CAMPOS & 1,05 &, 17 & 2,78 & 8,75 & 5,43 & 5,27 & 5,55 & 2,27 & 2,73 & 3,31 & 2,59 & 16,57 \\
\hline TAUBATÉ & 1,26 &, 24 & 5,01 & 8,50 & 6,20 & 8,18 & 6,28 & 2,32 & 2,42 & 3,19 & 1,29 & 17,88 \\
\hline MÉDIA GRUPO & 1,81 & 43 & 3,56 & 9,09 & 7,22 & 6,48 & 6,84 & 2,80 & 2,72 & 2,55 & 1,33 & 16,92 \\
\hline 5 BARUERI & 2,44 & ,59 & 4,19 & 8,19 & 9,68 & 8,93 & 8,81 & 3,68 & 4,68 & 3,24 & 5,45 & 20,85 \\
\hline CARAPICUIBA & 2,49 & ,66 & 3,73 & 8,34 & 7,86 & 6,09 & 6,34 & 3,33 & 3,32 & 1,46 & 4,31 & 17,49 \\
\hline COTIA & 1,86 & ,16 & 3,50 & 11,32 & 8,86 & 9,37 & 8,92 & 4,76 & 3,80 & 2,56 & 2,44 & 17,77 \\
\hline DIADEMA & 2,24 & ,37 & 3,50 & 12,07 & 8,95 & 5,02 & 7,02 & 4,13 & 3,52 & 3,63 & 7,85 & 17,36 \\
\hline EMBU & 2,26 & ,61 & 4,15 & 9,55 & 6,07 & 6,46 & 11,70 & 3,52 & 4,12 & 1,94 & 7,39 & 20,25 \\
\hline GUARULHOS & 2,13 &, 97 & 5,47 & 8,36 & 7,00 & 10,33 & 6,40 & 3,08 & 4,09 & 2,41 & 4,34 & 17,21 \\
\hline MAUÁ & 2,08 & ,69 & 3,77 & 8,91 & 6,78 & 7,16 & 7,42 & 4,61 & 2,60 & 1,68 & 4,70 & 19,82 \\
\hline OSASCO & 2,19 & ,64 & 4,66 & 9,33 & 6,56 & 8,17 & 6,56 & 3,15 & 3,24 & 1,89 & 5,47 & 18,52 \\
\hline PRAIA GRANDE & 2,41 &, 93 & 4,88 & 9,18 & 5,98 & 5,91 & 5,06 & 3,73 & 4,04 & 2,25 & 3,26 & 18,10 \\
\hline SĀO VICENTE & 2,54 & ,40 & 3,55 & 9,21 & 5,99 & 5,40 & 5,73 & 3,31 & 3,22 & 1,43 & $3, \mathbf{4 4}$ & 16,67 \\
\hline SUMARE * & 1,30 & 41 & $3, \mathbf{3 3}$ & 8,61 & 5,56 & 5,16 & 6,92 & 2,99 & 3,75 & 2,10 & 3,20 & 14,34 \\
\hline SUZANO & 2,76 &, 51 & 3,54 & 7,55 & 9,66 & 6,73 & 10,18 & 7,41 & 4,68 & 3,07 & 3,03 & 18,00 \\
\hline TABOĀO DA SERRA & 2,36 &, 43 & 3,52 & 13,50 & 6,52 & 7,25 & 7,61 & 2,76 & 4,58 & 2,58 & 5,00 & 18,71 \\
\hline MÉDIA GRUPO & 2,24 &, 57 & 3,98 & 9,55 & 7,34 & 7,08 & 7,59 & 3,88 & 3,82 & 2,33 & 4,61 & 18,08 \\
\hline 6 GUARUJÁ & 2,86 &, 70 & 3,30 & 8,81 & 3,94 & 9,74 & 10,46 & 3,36 & 3,64 & 1,26 & 3,25 & 18,87 \\
\hline 7 ITAPEVI & 2,70 & 21 & $\mathbf{4}, 78$ & 8,69 & 10,43 & 5,25 & 9,38 & 4,10 & 4,79 & 1,80 & 5,77 & 21,48 \\
\hline ITAQUAQUECETUBA. & 2,21 &, 57 & 4,10 & 8,21 & 9,72 & 6,08 & 10,43 & 3,84 & 4,22 & 2,31 & 5,86 & 16,49 \\
\hline MÉDIA GRUPO & 2,45 & 89 & $\mathbf{4 , 4 4}$ & 8,45 & 10,07 & 5,67 & 9,91 & 3,97 & 4,51 & 2,06 & 5,82 & 18,99 \\
\hline
\end{tabular}

*Divergentes ** Coeficiente por 10.000 habitantes

Fonte: Fundação SEADE, 1992

IESUS, VI(2), Abr/Jun, 1997. 
Quadro III - Qualificação dos grupos quanto aos indicadores sócio-econômicos e situação de saúde

\begin{tabular}{|c|c|}
\hline Indicadores sócio-econômicos & Indicadores de saúde \\
\hline $\begin{array}{l}\text { GRUPO } 1 \text { - predomínio nos índices de } \\
\text { analfabetismo > } 15 \text { anos, relação de } \\
\text { matrículas do } 2 \circ / 1 \circ \text { grau, renda em salários } \\
\text { mínimos e habitantes por domicílio. } \\
\text { Destaque positivo em outros } 5 \text { índices: água } \\
\text { inadequada, cômodos, crianças analfabetas, } \\
\text { leitos e pobreza. }\end{array}$ & $\begin{array}{l}\text { Baixos: homicídios e outras mortes } \\
\text { violentas. } \\
\text { Altos: tumores e doenças isquêmicas. } \\
\text { Nenhum destaque ou predomínio. }\end{array}$ \\
\hline $\begin{array}{l}\text { GRUPO } 2 \text { - destaque positivo em } 8 \text { índices } \\
\text { sócio-econômicos: água inadequada, } \\
\text { analfabetismo > } 15 \text { anos, crianças } \\
\text { analfabetas, pobreza, habitantes por } \\
\text { domicílio, cômodos, renda em salários } \\
\text { mínimos, evasão escolar. }\end{array}$ & $\begin{array}{l}\text { Baixos: homicídios e outras mortes } \\
\text { violentas. } \\
\text { Todos os outros com índices baixos, com } \\
\text { destaque para outras doenças do sistema } \\
\text { circulatório e cerebrovasculares. }\end{array}$ \\
\hline $\begin{array}{l}\text { GRUPO } 3 \text { - destaque positivo em } 3 \text { índices: } \\
\text { reprovação e relação de matrículas do } 2 \% / 1^{\circ} \\
\text { grau e cômodos. Índices ruins em evasão } \\
\text { escolar e pobreza. }\end{array}$ & $\begin{array}{l}\text { Baixos: homicídios e outras mortes } \\
\text { violentas. } \\
\text { Destaque para baixos índices em doenças } \\
\text { isquêmicas do coração e pneumonia. }\end{array}$ \\
\hline $\begin{array}{l}\text { GRUPO } 4 \text { - destaque positivo em } 3 \text { índices: } \\
\text { água inadequada, analfabetismo em }>15 \\
\text { anos e cômodos. Índices ruins em matrícula } \\
\text { na pré-escola e pobreza. }\end{array}$ & $\begin{array}{l}\text { Baixos: homicídios e outras mortes } \\
\text { violentas. } \\
\text { Todos os outros coeficientes com baixos } \\
\text { índices. } \\
\text { Nenhum destaque ou predomínio. }\end{array}$ \\
\hline $\begin{array}{l}\text { GRUPO } 5 \text { - destaque negativo em } 6 \text { índices: } \\
\text { esgoto inadequado, leitos, domicílios com } 2 \\
\text { ou } 3 \text { cômodos, reprovação, matrícula na pré- } \\
\text { escola e relação de matrículas do } 2 \% / 1^{\circ} \text { grau. }\end{array}$ & $\begin{array}{l}\text { Altos: homicídios e outras mortes violentas. } \\
\text { Todos os outros com índices altos, com } \\
\text { destaque em perinatais. }\end{array}$ \\
\hline $\begin{array}{l}\text { GRUPO } 6 \text { - piores índices de reprovação e } \\
\text { de crianças analfabetas, destaca-se } \\
\text { negativamente também em } 6 \text { outros } \\
\text { indicadores: cômodos, domicílio com } 2 \text { ou } 3 \\
\text { cômodos, relação de matrículas do } 2^{\circ} / 1^{\circ} \\
\text { grau, analfabetismo em > } 15 \text { anos, } \\
\text { matrícula na pré-escola e pobreza. }\end{array}$ & $\begin{array}{l}\text { Altos: homicídios e outras mortes violentas. } \\
\text { Índices altos em isquêmicas do coração e } \\
\text { cerebrovasculares. }\end{array}$ \\
\hline $\begin{array}{l}\text { GRUPO } 7 \text { - destaque para os piores índices } \\
\text { de lixo e esgoto, predominância negativa } \\
\text { também em } 11 \text { outros indicadores: água, } \\
\text { analfabetismo em > } 15 \text { anos, crianças } \\
\text { analfabetas, relação de matrículas do } 2^{\circ} / 1^{\circ} \\
\text { grau, matrículas na pré-escola, cômodos, } \\
\text { domicílio com } 2 \text { ou } 3 \text { cômodos, habitantes } \\
\text { por domićlio, leitos, pobreza e renda em } \\
\text { salários mínimos. }\end{array}$ & $\begin{array}{l}\text { Altos: homicídios e outras mortes violentas. } \\
\text { Destaque negativo para o maior índice em } \\
\text { outras doenças do sistema circulatório, } \\
\text { predominância negativa em } \\
\text { cerebrovasculares e enterites. }\end{array}$ \\
\hline
\end{tabular}


Mortalidade em Municípios de SP e Indicadores Sócio-econômicos: uma Análise de Agrupamento

Tabela IV - Relação dos municípios que divergem em indicadores de saúde dos grupos originados pela análise de agrupamento

\begin{tabular}{|c|c|c|c|c|}
\hline MUNICÍPIO & $\begin{array}{c}\text { GRUPO } \\
\text { OBSERVADO } \\
\text { (AGRUPAM) }\end{array}$ & $\begin{array}{c}\text { GRUPO } \\
\text { PREDITO } \\
\text { (DISCRIM) }\end{array}$ & $\begin{array}{c}\text { PROBABILIDADE } \\
A^{*}\end{array}$ & $\begin{array}{l}\text { PROBABILIDADE } \\
\mathrm{D}^{* *}\end{array}$ \\
\hline BAURU & 2 & 3 & $10,18 \%$ & $81,58 \%$ \\
\hline RIO CLARO & 2 & 3 & -- & $92,49 \%$ \\
\hline SAO CARLOS & 2 & 4 & $40,22 \%$ & $40,64 \%$ \\
\hline INDAIATUBA & 4 & 2 & $41,00 \%$ & $58,79 \%$ \\
\hline PIRACICABA & 4 & 2 & $43,15 \%$ & $5.2,57 \%$ \\
\hline SANTA BÁRBARA & 4 & 3 & -- & $43,52 \%$ \\
\hline D'OESTE & & & & \\
\hline SĀO JOSÉ DOS CAMPOS & 4 & 2 & $24,66 \%$ & $74,00 \%$ \\
\hline SUMARÉ & 5 & 2 & $43,98 \%$ & $50,98 \%$ \\
\hline
\end{tabular}

- Probabilidade do municipio pertencer ao grupo observado (Análise de Agrupamento)

** Probabilidade do municipio pertencer ao grupo predito (Análise Discriminante) 